\title{
The role of ultrasonography in the diagnostic criteria for rheumatoid arthritis and monitoring its therapeutic efficacy
}

\author{
Sławomir Jeka ${ }^{1, A, D-F}$, Marta Dura ${ }^{3,5, A, B}$, Paweł Zuchowski, ${ }^{2, A-D}$, Beata Zwierko ${ }^{3, B}$, Marzena Waszczak-Jeka 4,B \\ ${ }^{1}$ Clinic of Rheumatology and Systemic Connective Tissue Disorders, Jan Biziel University Hospital No. 2, Ludwik Rydygier Collegium Medicum in Bydgoszcz, UMK in Toruń, Poland \\ ${ }^{2}$ Clinic of Rheumatology and Systemic Connective Tissue Disorders, Jan Biziel University Hospital No. 2, Bydgoszcz, Poland \\ ${ }^{3}$ Department of Radiology, Jan Biziel University Hospital No. 2, Bydgoszcz, Poland \\ ${ }^{4}$ Centre for Clinical Trials, Warszawa, Poland \\ ${ }^{5}$ Ludwik Rydygier Collegium Medicum in Bydgoszcz, Nicolaus Copernicus University, Toruń, Poland
}

A - research concept and design; $\mathrm{B}$ - collection and/or assembly of data; $\mathrm{C}$ - data analysis and interpretation;

$D$ - writing the article; $E$ - critical revision of the article; $F$ - final approval of the article

Address for correspondence

PawełZuchowski

E-mail: p.zuchowski81@gmail.com

Funding sources

None declared

Conflict of interest

None declared

Received on December 28, 2016

Reviewed on January 25, 2017

Accepted on February 23, 2017

\begin{abstract}
Rheumatoid arthritis (RA) is a chronic systemic disease of connective tissue. It is characterized by symmetrical multiple joint involvement and extra-articular symptoms. Modern RA treatment methods place a particular emphasis on the earliest possible diagnosis and initiation of appropriate treatment. Currently, ultrasonography (US) is the key imaging test performed in RA patients. However, despite the general acknowledgement of its role in the assessment of disease activity, US was not included in the applicable ACR/EULAR criteria. This is due to the lack of strictly defined criteria for US evaluation and the interpretation of test results. In addition, the absence of a correlation between the common DAS/DAS28 disease activity score and ultrasound assessment of joints makes developing new diagnostic criteria difficult. The objective of this article is to review recent scientific reports on the use of ultrasonography in the diagnosis and monitoring of RA and to indicate current problems associated with the interpretation of test results and the comparison with applicable scores of disease activity.
\end{abstract}

Key words: rheumatoid arthritis, ultrasonography, synovitis

DOI

10.17219/acem/69133

Copyright

Copyright by Author(s)

This is an article distributed under the terms of the

Creative Commons Attribution Non-Commercial License

(http://creativecommons.org/licenses/by-nc-nd/4.0/) 


\section{Introduction}

Rheumatoid arthritis (RA) is a chronic systemic disease of connective tissue. The most characteristic symptom of RA is non-specific inflammation of symmetric joints. In early rheumatoid arthritis (ERA), inflammatory lesions usually affect the joints of the hands and feet. Currently, it is believed that inflammation of the joint synovial membrane is the most valid indicator of the course of the disease.

Nowadays, RA treatment methods focus on the earliest possible diagnosis and initiation of appropriate treatment. Treatment is aimed at reducing pain and improving the patient's quality of life by minimizing or completely blocking inflammatory processes in the joint synovium. Such therapy delays radiological progression, i.e., joint damage, and thus prevents the patient's disability level from worsening.

The earlier effective treatment is initiated, the better the prognosis. Unfortunately, early RA diagnosis is challenging due to its non-specific onset.

The 2010 American College of Rheumatology/European League Against Rheumatism criteria (ACR/EULAR) for RA currently in use are much more sensitive and specific than the previous 1987 version. However, they still cannot be considered complete as further amendments may be anticipated. The sensitivity of the current classification criteria is between $58 \%$ and $91 \%$ in patients with RA lasting for less than 2 years. ${ }^{1}$ However, the detectability of RA in cases of shorter disease duration is much lower. In patients with RA lasting for less than 3 months (which can be considered ERA), the sensitivity is $62-74 \% .{ }^{1}$ Consequently, 1 in 4 ERA patients remains undiagnosed during the $1^{\text {st }}$ few weeks of the disease. As a result, treatment initiation is delayed, and the patient's chances for maintaining full physical function and achieving sustained remission are lower.

Delayed treatment is also associated with poorer prognosis and a higher risk of complications. The most common consequences of delaying therapy include joint damage, a higher risk of orthopedic surgery at a further stage of the disease, multiple organ complications, and increased mortality.

Although it is hard to prove that there is indeed such a window of opportunity, it is certain that early RA diagnosis and administration of appropriate treatment with synthetic disease-modifying antirheumatic drugs (sDMARDs) and biological DMARDs (bDMARDs) increase the chances of remission. ${ }^{2,3}$

Consequently, the improvement of the current RA classification criteria to increase diagnostic sensitivity to early RA is crucial.

\section{Imaging diagnostics}

According to many rheumatologists and radiologists, modern imaging diagnostic tests, i.e., ultrasonography (US) and magnetic resonance imaging (MRI), are particularly significant for RA diagnostics. In the case of ERA, the diagnostic applicability of X-rays is much lower.

Of course, X-rays were included in the RA classification criteria as early as the Rome 1961 criteria. ${ }^{4}$ Obviously, this should be considered in the historical context, as until the 1990s, US and MRI tests were relatively novel imaging techniques and the image quality they offered was inferior to that obtained today. For the same reason, the revised 1987 RA classification criteria did not take the methods into account either. ${ }^{4}$

Only during the last 20 years has a remarkable change taken place in the recognition of imaging tests as a diagnostic tool. Significant developments with respect to US, MRI, and computed tomography (CT) have granted imaging diagnostics a prominent position in rheumatology.

Computed tomography is not commonly used despite the fact that it is especially useful for bone lesion imaging. ${ }^{5}$ This is due to the high amount of ionizing radiation the patients are exposed to during the test.

Magnetic resonance imaging, because of its long duration and high costs, has not become a basic RA diagnostic tool either. However, it is performed much more frequently than CT and its spectrum of applicability to RA diagnostics is broad. ${ }^{6,7}$

Ultrasonography is the basic imaging test used in RA diagnostics. In contrast to MRI, it is easily accessible and cost-effective.

\section{Ultrasonography: The current status}

Over the last few decades, US has gradually become more and more significant in RA diagnostics, which resulted in the establishment of the OMERACT initiative in $2004 .{ }^{5,8}$ The objective of this group is to define RA diagnostic criteria in US tests. Although the initiative has existed for many years, no consensus or consistent principles on assessing US results in RA patients have been developed, and no decision on which joints should be evaluated has been made..$^{5,8}$

Discrepancies in the evaluation of US findings resulted in the technique being omitted from the latest 2010 ACR/ EULAR criteria. However, it should be emphasized that both ACR and EULAR recognize the significance of US in ERA and RA diagnosis and that neither of the organizations question its applicability. They established expert teams in order to work on introducing US to future RA classification criteria.

Despite the fact that US is not currently included either in the classification criteria or in standard disease activity scores or therapeutic efficacy measures - such as DAS/DAS28 - it is commonly performed by rheumatologists. ${ }^{9}$

Ultrasonography with a high-frequency $(15-18 \mathrm{MHz})$ linear array transducer probe enables the assessment of early lesions in small joints and the detection of inflammatory changes in the synovial membrane. 
Table 1. Joint inflammation assessment based on PDUS ${ }^{10}$

\begin{tabular}{|c|c|}
\hline Grade & Description \\
\hline 0 & normal (no PD signal) \\
\hline 1 & $\begin{array}{c}\text { mild hyperaemia (PD signal covers up to } 25 \% \text { of the } \\
\text { synovium) }\end{array}$ \\
\hline 2 & $\begin{array}{c}\text { moderate hyperaemia (PD signal covers from }>25 \% \text { up } \\
\text { to } 50 \% \text { of the synovium) }\end{array}$ \\
\hline 3 & $\begin{array}{c}\text { marked hyperaemia (PD signal covers more than } 50 \% \text { of the } \\
\text { synovium) }\end{array}$ \\
\hline
\end{tabular}

PDUS - power Doppler ultrasonography.

Where power Doppler ultrasonography (PDUS) is used, blood flow within the joint synovium can be assessed, and consequently, inflammatory processes can be observed and evaluated (Table 1). ${ }^{10}$

\section{Ultrasonography: Diagnostic challenges}

Despite its broad use in RA diagnosis and monitoring, US poses a number of problems. Apart from the lack of consensus on which joints should be evaluated, the subjectivity inherent in US interpretation cannot be disregarded.

Erosions are one of the most common symptoms associated with RA, apart from synovitis, which can be observed in US. The prevention of erosions is one of the aims of RA treatment. Their development in RA patients is considered an indicator of poor prognosis for the further course of the disease. Consequently, their detection and on-treatment assessment may have a significant impact on decisions concerning therapy.

The correct assessment of erosions in US is not always unambiguous. The minimum size of an erosion is currently a topic of debate. ${ }^{5}$ Along with the higher frequencies provided by transducers, images of increasingly higher resolutions can be obtained. This means that smaller structures can be visualized, which may lead to an overdiagnosis of erosions. Recent studies demonstrate that erosion-like lesions may also be detected in healthy individuals. ${ }^{11}$ As a result, a minimum erosion size would be an essential criterion in the diagnostic process. Currently, it is believed that the minimum size should be at least $2 \mathrm{~mm} .^{5}$

The monitoring of erosion changes over the course of the disease is also a problem. Unfortunately, the simplest solution in this case, i.e., counting the erosions, is not a valid criterion for the assessment of disease progression. ${ }^{5} \mathrm{With}$ such an approach, cases involving the merging of 2 erosions can be challenging. In addition, the assessment of erosion activity by PDUS is necessary.

Researchers agree as to which joints should be included in erosion assessment. The metacarpophalangeal joints (MCP) and the metatarsophalangeal joints (MTP) have been suggested for assessment. ${ }^{5}$

Apart from erosions, synovitis is the most characteristic sign of RA. Its assessability through US/PDUS is one of the main reasons for the common use of ultrasonography in monitoring disease progression.

Many scientific and clinical studies have evaluated the efficacy of methotrexate monotherapy (MTX) or a combination therapy with MTX + anti-TNF inhibitors or biologics with a different mechanism of action, based not only on the activity scores (DAS/DAS28) but also on synovitis.

One of the reasons why such an emphasis is put on introducing US/PDUS to treatment efficacy assessment is the fact that remission with respect to DAS/DAS28 has been observed in patients despite the presence of synovitis and the resulting progression of radiological changes. ${ }^{12,13}$

Power Doppler ultrasonography enables the blood flow in the synovium of small joints in the hands and feet to be assessed. There is a correlation between PDUS results and laboratory test findings. ${ }^{14}$ In addition, the sensitivity of PDUS is higher than that of physical examination. ${ }^{14}$

Novel treatment options, i.e., biologics, entail modifications not only in the modality of therapy, but also in the monitoring of its effects. Targeted treatment (treat-totarget, T2T) and recent EULAR guidelines recommend changing the treatment strategy after 3 months if the therapy proves ineffective or after 6 months if the treatment target has not been reached. ${ }^{15-17}$ Such an approach to treatment also requires adopting a new approach to the assessment of its efficacy. In line with the current knowledge of the essence of RA as a disease causing inflammation of the synovial membrane, its assessment in PDUS should be best suited to reflect the efficacy of the therapy.

\section{RA activity assessment on the basis of PDUS and DAS: Differences}

DAS was developed with a view to assessing ERA patients. ${ }^{18}$ At present, it is a standard score used in patients with RA lasting for many years. At the time of its development, imaging tests (US, CT and MRI) did not provide images of such a high quality as the ones available today. For this reason, DAS authors did not even consider including imaging diagnostics in the score.

Most commonly, over the course of RA, irreversible destructive joint lesions develop. In such cases, joint pain is no longer associated with disease activity. In this situation, despite improvements in the patient's health and the absence of synovitis in US/PDUS results, according to DAS still no remission occurs. This is connected with the significant impact of the number of tender joints on the total DAS result. ${ }^{18}$ On the other hand, situations have been reported where US/PDUS scores indicate the presence of inflammation in joints which presents no symptoms in the physical examination, i.e., the patient does not feel pain and the assessing physician does not find any evidence of joint swelling. 
Owing to the above situations, US/PDUS tests are not an appropriate representation of changes in DAS, which has been confirmed in scientific and clinical studies.

In a study conducted with the participation of 102 patients in remission (DAS28 <2.6), a 1 -year progression in radiological changes detected in PDUS and MRI was confirmed in 19 patients (19\%). ${ }^{13}$

In another study with 149 subjects, no positive correlation between the currently applicable remission criteria (ACR/EULAR 2010) and PDUS results was demonstrated. ${ }^{19}$ In almost every other patient who met the remission criteria, PDUS scores confirmed the presence of synovitis. ${ }^{19}$ A group of patients with no synovitis revealed in PDUS was also large despite the fact that they were not in remission according to the currently applicable criteria. ${ }^{19}$ From among the parameters taken into consideration in the 2010 ACR/EULAR remission criteria, only C-reactive protein (CRP) and morning stiffness duration were correlated with the US/PDUS findings. ${ }^{19}$ According to the authors, the parameters included in the assessment criteria for disease activity are often very subjective, which makes it difficult to achieve a correlation with US/PDUS results characterized by higher objectivity and repeatability. ${ }^{19}$

Saleem et al. suggest that currently DAS28 is not the best measure of remission assessment. ${ }^{20}$ A DAS 28 of $<2.6$ does not exclude the presence of tender and swollen joints or increased CRP and erythrocyte sedimentation rate (ESR) values, although it represents remission. Their study enrolled 128 patients in remission (DAS28 $<2.6$ ) in whom no flare-ups had been observed during 6 months preceding the study enrolment and treatment had not been modified during that period. The patients received DMARDs $(n=66)$ or MTX + anti-TNF $(n=62)$. In 40 patients $(31 \%)$, physical examination revealed the swelling of at least one joint although these patients were in DAS28 remission. ${ }^{20}$ Based on PDUS scores, these patients were diagnosed with synovitis. PDUS results were much better correlated with the clinical assessment based on the Simplified Disease Activity Score (SDAI). ${ }^{20}$ The authors suggest that the current remission criteria are more indicative of low disease activity than actual remission, and would better correlate with a US/PDUS test if the criteria were more restrictive. ${ }^{20}$

An interesting analysis of US applicability in remission assessment in RA patients was performed by Balsa et al. ${ }^{21}$ They studied a group of 97 patients who received treatment with DMARDs or biologics. Remission was assessed using the 2 most common scores: the DAS28 and the SDAI. In addition, US/PDUS of 42 joints (proximal interphalangeal joints, MCPs, radiocarpal joints, radioulnar joints, glenohumeral joints, knee joints, ankle joints, metatarsal joints and MTPs) was performed. The joints' ultrasound evaluation was performed on the basis of the OMERACT score. In the study group, synovial overgrowth was found in 92 patients (95\%), whereas a PD signal was observed in the case of 41 individuals (42\%). DAS28 remission was confirmed in 74 subjects (76\%), while according to SDAI criteria, remission was confirmed in 54 patients (56\%). As compared with US findings, SDAI remission assessment is more accurate than that based on the DAS28. The study demonstrated that both SDAI and DAS28 scores render similar results in patients with active disease, whereas in the case of remission, significant discrepancies between the 2 measures can be observed. ${ }^{21}$

The APPRAISE study conclusions completely undermine the reasonability of comparing the results of US/ PDUS with the DAS28. ${ }^{15}$ This study enrolled biologicnaive patients with insufficient improvement from MTX therapy. During this 24-week study, abatacept was administered to all patients. During the study, PDUS tests of 44 joints and DAS28 assessment were performed in patients on a regular basis. PDUS results were assessed using the Global OMERACT-EULAR Synovitis Score (GLOESS). At the completion of the study, a total absence of correlation between disease activity assessments with PDUS and the DAS28 was demonstrated. ${ }^{15}$

These observations are consistent with previously presented studies, and they also suggest a higher correlation between PDUS and SDAI findings. ${ }^{15}$

The authors explain the lack of consistency between PDUS and the DAS28 by the fact that PDUS enables the detection of much smaller lesions than is possible with joint physical examination. ${ }^{15}$

The thesis seems to be confirmed by another study in which PDUS results were compared with the Clinical Disease Activity Index (CDAI) scores. A strong correlation was revealed only with respect to grade 3 synovitis on the basis of PDUS and CDAI evaluations. ${ }^{22}$

\section{PDUS-assessed joints}

In clinical practice, PDUS with multiple joints assessment is rarely performed due to timing considerations. ${ }^{23}$ Usually, even 6-joint evaluation may pose a significant challenge due to the duration of the patients' visits. Consequently, doctors search for joints in which inflammatory lesions would be most reflective of the course of RA. Grassi et al. suggest that assessing a single joint may be sufficient for the assessment of disease activity and therapeutic efficacy. ${ }^{23}$ According to them, MCP lesions are most indicative of the disease status.

Considering the discrepancies with regard to the number of joints assessed by individual researchers, attention should be paid to the results of the study conducted by Yoshimi et al. ${ }^{24}$ They demonstrated that changing the number of assessed joints (24 or 8 ) did not have a significant impact on the assessment of RA activity.

It suggests that RA activity assessment does not, in fact, require a comprehensive US/PDUS test and that assessing only a few joints provides accurate disease status results. 


\section{Conclusions and future considerations}

The results of recent studies show that both US/PDUS and the DAS/DAS28 are valid indicators of the patient's health improvements. However, looking for a correlation between them is wrong, as they reflect different aspects of the disease. ${ }^{15}$ SDAI and CDAI scores are much better correlated with US/PDUS than DAS28 due to their higher objectivity, since the health status is assessed by the physician.

PDUS signal assessment depends on the operator. If the pressure of the transducer probe on the evaluated joint is excessive, the values can be easily distorted. ${ }^{18}$ For this reason, training courses for operators need to be introduced in order to improve the quality of the testing. ${ }^{25} \mathrm{Cer}-$ tainly, skilful test performance should not be particularly difficult; therefore, the issue is not likely to be an obstacle in the introduction of PDUS to the classification criteria. ${ }^{19}$

The advances in imaging diagnostics seen over the last 2 decades have had a significant impact on RA patient management. Currently, treatment without CT, MRI, or US is hard to imagine. Among these tests, US has become the most popular one because of its low costs and short duration of testing.

At present, the value of US/PDUS, both in diagnostics and in the monitoring of therapeutic efficacy, is indisputable. There is a shared conviction that the test should be introduced to the classification criteria. However, despite the publication of the results of many studies, the number of joints which need to be assessed is still being discussed. Comparing PDUS scores with the DAS, which was developed almost 40 years ago and has not changed much since then, can also be challenging. Recently, authors have been indicating that according to the current knowledge, DAS is not a correct measure of the disease and that juxtaposing it with US/PDUS synovial assessment is pointless. ${ }^{15}$

Potentially, US/PDUS and DAS/DAS28 scores should be no longer compared. Applicability of US is generally acknowledged, and discrepancies between the test results and the DAS/DAS28 assessment are well documented. At present, more attention should be paid to selecting joints for assessment as part of routine rheumatologic examinations and to developing clear criteria for the evaluation of joints.

\section{References}

1. Migliore A, Bizzi E, Petrella L, et al. The challenge of treating earlystage rheumatoid arthritis: The contribution of mixed treatment comparison to choosing appropriate biologic agents. BioDrugs. 2016; 30:105-115.

2. van Nies JA, Tsonaka R, Gaujoux-Viala C, et al. Evaluating relationships between symptom duration and persistence of rheumatoid arthritis: Does a window of opportunity exist? Results on the Leiden early arthritis clinic and ESPOIR cohorts. Ann Rheum Dis. 2015;74:806-812.

3. Świerkot J, Batko B, ed. Reumatoidalne zapalenie stawów: praktyczne aspekty leczenia. $1^{\text {st }}$ ed. Poznań, Poland: Termedia Wydawnictwa Medyczne; 2016.
4. Arnett FC, Edworthy SM, Bloch DA, et al. The American Rheumatism Association 1987 revised criteria for the classification of rheumatoid arthritis. Arthritis Rheum. 1988;31:315-324.

5. Szkudlarek M, Terslev L, Wakefield RJ, et al. Summary findings of a systematic literature review of the ultrasound assessment of bone erosions in rheumatoid arthritis. J Rheumatol. 2016;43:12-21.

6. Boutry N, Morel M, Flipo RM, et al. Early rheumatoid arthritis: A review of MRI and sonographic findings. Am J Roentgenol. 2007;189: 1502-1509.

7. Piotto DG, Correa MJ, Miotto e Silva VB, et al. Laser Doppler imaging for assessment of microcirculation in juvenile systemic sclerosis. Rheumatology (Oxford). 2014;53:72-75.

8. Naredo E, Wakefield RJ, lagnocco A, et al. The OMERACT ultrasound task force-status and perspectives. J Rheumatol. 2011;38:2063-2067.

9. Naredo E, lagnocco A. Why use ultrasound in rheumatology? Rheumatology (Oxford). 2012;51(Suppl 7):vii1.

10. Fiocco U, Ferro F, Vezzù M, et al. Rheumatoid and psoriatic knee synovitis: Clinical, grey scale, and power Doppler ultrasound assessment of the response to etanercept. Ann Rheum Dis. 2005;64:899-905.

11. Finzel $S$, Ohrndorf $S$, Englbrecht $M$, et al. A detailed comparative study of high-resolution ultrasound and micro-computed tomography for detection of arthritic bone erosions. Arthritis Rheum. 2011;63: 1231-1236.

12. Brown AK, Quinn MA, Karim Z, et al. Presence of significant synovitis in rheumatoid arthritis patients with disease modifying antirheumatic drug-induced clinical remission: Evidence from an imaging study may explain structural progression. Arthritis Rheum. 2006;54: 3761-3773.

13. Brown AK, Conaghan PG, Karim Z, et al. An explanation for the apparent dissociation between clinical remission and continued structural deterioration in rheumatoid arthritis. Arthritis Rheum. 2008;58: 2958-2967.

14. Sreerangaiah D, Grayer M, Fisher BA, et al. Quantitative power Doppler ultrasound measures of peripheral joint synovitis in poor prognosis early rheumatoid arthritis predict radiographic progression. Rheumatology (Oxford). 2016;55:89-93.

15. D'Agostino MA, Boers M, Wakefield RJ, et al. Exploring a new ultrasound score as a clinical predictive tool in patients with rheumatoid arthritis starting abatacept: Results from the APPRAISE study. RMD Open. 2016;5,2:e000237.

16. Smolen JS, Aletaha D, Bijlsma JW, et al. Treating rheumatoid arthritis to target: Recommendations of an international task force. Ann Rheum Dis. 2010;69:631-637.

17. Smolen JS, Breedveld FC, Burmester GR, et al. Treating rheumatoid arthritis to target: 2014 update of the recommendations of an international task force. Ann Rheum Dis. 2016;75:3-15.

18. Barczyńska TA, Dura M, Blumfield E, et al. DAS28 score vs ultrasound examination for assessment of rheumatoid arthritis disease activity: Comparison and discussion of pros and cons. Reumatologia. 2015; 4:213-218.

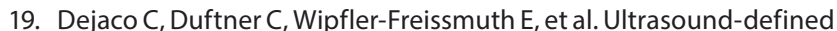
remission and active disease in rheumatoid arthritis: Association with clinical and serologic parameters. Semin Arthritis Rheum. 2012;41: 761-767.

20. Saleem B, Brown AK, Keen $\mathrm{H}$, et al. Should imaging be a component of rheumatoid arthritis remission criteria? A comparison between traditional and modified composite remission scores and imaging assessments. Ann Rheum Dis. 2011;70:792-798.

21. Balsa A, de Miguel E, Castillo C, et al. Superiority of SDAI over DAS-28 in assessment of remission in rheumatoid arthritis patients using power Doppler ultrasonography as a gold standard. Rheumatology (Oxford). 2010;49:683-690.

22. Gärtner $M$, Mandl $P$, Radner $H$, et al. Sonographic joint assessment in rheumatoid arthritis: Associations with clinical joint assessment during a state of remission. Arthritis Rheum. 2013;65:2005-2014.

23. Grassi W, Gaywood I, Pande I, et al. From DAS 28 to SAS 1. Clin Exp Rheumatol. 2012;30:649-651.

24. Yoshimi R, Ihata A, Kunishita Y, et al. A novel 8-joint ultrasound score is useful in daily practice for rheumatoid arthritis. Mod Rheumatol. 2015;25:379-385. 